\title{
Application of Product Data Manage- ment Technology for Enterprise-wide Distributed Product Development
}

Prof. Dr.-Ing. M. Abramovici,

Dipl.-Ing. D. Gerhard,

Dipl.-Ing. L. Langenberg

Information Technology in Mechanical Engineering (ITM)

Ruhr-Universitaet Bochum

D-44780 Bochum, Germany

Tel.: $\quad+492347007009$

Fax: $\quad+492347094443$

e-mail: \{abr,dege,lars\}@itm.ruhr-uni-bochum.de

\begin{abstract}
The paper gives a comprehensive overview and a classification of the fundamentals and the current state of the art of Product Data Management (PDM) technology regarding the support of distributed product development. Based on experiences and results of actual industrial projects, the requirements for distributed product development are presented. Considering these demands, different application approaches for building a distributed PDM solution using commercially available systems as well as methods for the replication and exchange of product data under consideration of existing standards are introduced and evaluated. Finally the demands for further developments concerning tools and methods, and standardisation are addressed and a new research approach of ITM, Bochum with special respect to flexibility and ease of implementation is shown.
\end{abstract}

\section{Keywords}

PDM, EDM, CAD, CSCW, Life Cycle Management, Information Technology, Distributed Product Development 


\section{INTRODUCTION}

Growing global competition as well as new regulations and laws concerning environmental issues, quality issues, etc. have lead to dramatic structural and technological changes within industry. Furthermore, the majority of today's industrial products has an increased complexity including mechanical components, electronic hardware, software and value added services. Co-operative product development within cross-functional, globally dispersed design teams is one means to maintain competitiveness. This leads to tasks like enterprise wide sharing and co-ordination of product and process data across engineering boundaries, collaborative release and change management. On the other hand emerging technologies like Internet or engineering marketplaces (Figure 1) offer additional opportunities for co-operation but also require extensive use of advanced information technology.

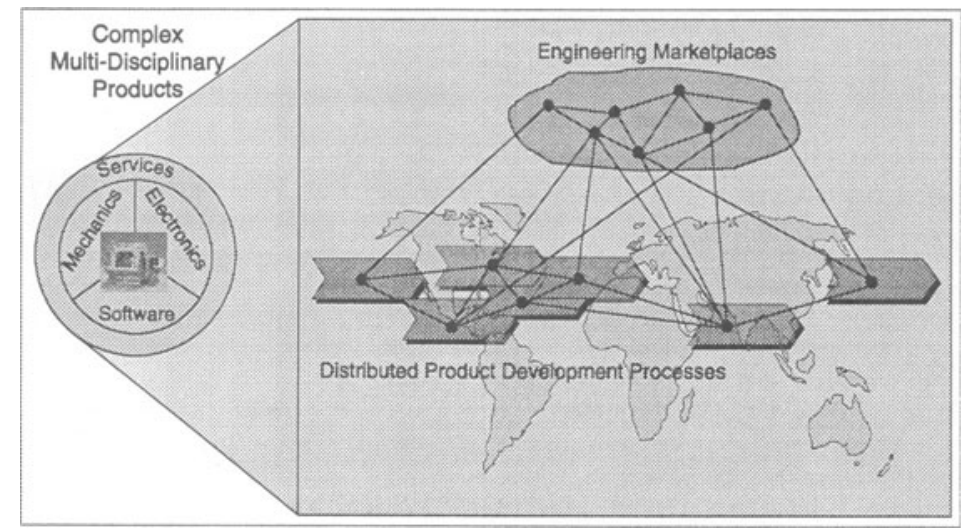

Figure 1 Distributed product development processes for complex products

PDM technology, which this article is focusing on, offers general capabilities for managing and sharing data and therefore is an appropriate approach to support co-operative product development teams. Most of the available PDM-tools, however, are not well suited for the support of distributed development activities, as they focus on homogeneous IT system environments. Homogeneous IT-system environment in this case denotes the use of a single PDM-system with a uniform corporate wide installation using one single internal data model. These are usually not available, however, as co-operation in general means heterogeneous multi-site environments with different semantics, legacy tools etc..

Since most co-operating companies, especially SME, generally do not have enough know-how and capacity to develop an appropriate solution on their own, commercially available tools have to be employed to support distributed product development processes. Thus the objective is to use a commercially available PDM-system in order to build an individual solution by customising, integrating legacy systems, and using available standards for data exchange, and to define 
methods, regulations, and instructions for co-operative use. In the following sections, available PDM-technology and applications of PDM for distributed product development, the potentials and benefits as well as the drawbacks, are described and the demands for further development of PDM technology are shown leading to new approaches for an optimal support of distributed multi-disciplinary processes.

\section{INDUSTRIAL REQUIREMENTS}

The common access of different partners to distributed engineering information is the main goal to be achieved by the PDM application in multi-site product development processes. Furthermore, the integration of heterogeneous information ressources for product development, e.g. engineering marketplaces, standard part libraries or patent information services, is gaining significant importance and has to be considered, too.

For the determination of industrial requirements, three scenarios of different types of co-operation, each indicating specific demands, have been analysed. Those scenarios are a multi-site networked enterprise, a customer-supplier-chain, and a virtual enterprise.

Within a multi-site networked enterprise different sites may be involved in product development but usually follow the same organisational concept. For instance release processes cover the same number of steps and intermediate states at each site of the enterprise. A data and process management solution for a networked enterprise has to support distributed workflows within either homogeneous or heterogeneous environments. Each site involved in product development has to access its own product data as well as product data of remote sites and, therefore, requires assistance for such tasks as exchange of model, metadata and organisational data, logging of each transfer, authentication and encryption.

All participants of a customer-supplier-chain remain independent but they are responsible for the development of specified subsystems of a product. The overall control of the product development is done by the customer. Therefore data usually is exchanged for the purpose of viewing and annotation, but is managed and changed at one single site. The development processes are loosely linked. Each supplier has to deliver defined results at fixed deadlines but can keep his own internal processes. As a result each participant keeps his specific IT infrastructure, organisation, data management and process management. These heterogeneous environments have to be harmonised during the constitution of a customersupplier-chain.

Within a virtual enterprise all partners act autonomously in order to achieve a common goal which is given and controlled by a central co-ordination instance. Similar to customer-supplier-chains the internal processes are independent of each other, but are subordinate to a common enterprise-spanning development process. Due to the dynamic and time-limited character of a virtual enterprise a fast 
constitution and dissolution is necessary. All partners are obliged to maintain their IT infrastructure, data and process management solutions and to rely on flexible, IT supported linking and interchange concepts. The main focus in the area of data exchange is the transfer of descriptive, classifying and organisational data whereas the exchange of model data requires additional assistance.

Within all these types of co-operation, a vertical and a horizontal integration has to be distinguished. Vertical in this particular case means co-operation within the same phase of the product life cycle whereas horizontal co-operation indicates different tasks along the product life cycle, for instance design, manufacturing, quality assurance, technical documentation, etc.. Accordingly, the functional requirements are different. They extend from information exchange on a read-only basis up to exchange or sharing of geometrical data and models. This leads, in addition to the IT-oriented technical feasibility within heterogeneous environments, to the following general demands concerning the functionality of distributed PDM solutions:

- quick and easy access, transfer, distribution and conversion of product data of each partner including organisational and structural data for direct further processing within data exchange processes

- controlled import and the export of data between different sites by acquisition, logging, acknowledgement and filing of inputs and outputs during data exchange, to guarantee the recherchability and the recoverability of access and exchange processes

- the ability of distributed data storage with a controlled redundancy and the capability of searching in distributed data bases

- low effort for customisation and configuration and the capability of an easy and stepwise integration into the existing legacy IT system landscape

- enabling of a cross-site workflow management to support and synchronise distributed product development processes, e.g. notification, release and change processes

- the ability to implement safety, access protection and encryption mechanisms of exchange data according to bilateral defined declarations to maintain security and authentication of confidential data

- fast transfer rates respectively short response times during cross-site actions

\section{PDM - STATE OF THE ART}

Basically PDM systems distinguish metadata which are managed in databases and bulk data stored on file system level. Metadata contains classifying respectively attributive information of objects, whereas bulk data indicates appendant files (CAD, text, graphic, etc.). Existing distributed PDM approaches consider homogeneous and heterogeneous IT environments and different levels of integration. Homogeneous means that the distributed PDM solution is based on a single system. In contrast to this, heterogeneous PDM environments have to combine more than one PDM system or even additional information resources like 
engineering marketplaces etc. The range of integration extends from loose connection of different PDM-sites up to a deep integration of PDM systems using one logical data model and uniform technical and administrative processes or different data models and set-ups. In the following sections distributed, linked and federated PDM approaches are examined closely.

\subsection{Distributed PDM}

Distributed PDM environments are built up from a main PDM server, optional sub servers and distributed clients, that are either directly or indirectly (via the sub server) connected to the main server leading to a hierarchical system structure. The bulk data can be distributed correspondingly over the different servers. The databases in which the metadata of the entities managed in the PDM system are held, may be distributed over the different servers, too. A central database implemented in general on the main server is used to avoid logic contradictions and to guarantee data integrity and data security. The individual sub servers with their databases are not autonomous but dependent on the main server, i.e. the entire system is damaged in the case of a loss of the main server. The overall system is spatially distributed but based upon a homogeneous PDM environment.

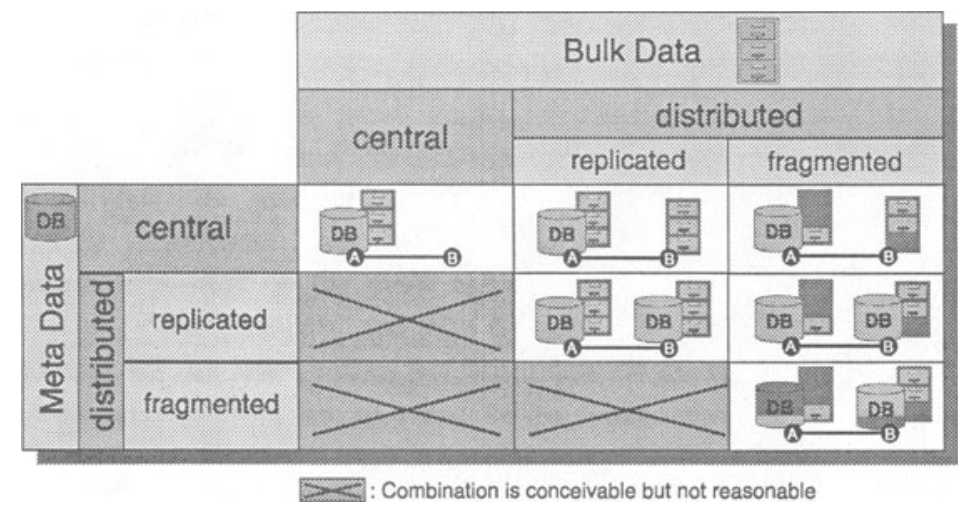

Figure 2 Classification of different approaches for distributed PDM

Figure 2 shows possible approaches for distributed PDM environments regarding the distribution of metadata and bulk data. Several approaches to distribute metadata and bulk data are conceivable but only a few are reasonable because of database or network limitations. For instance, considering a central PDM system approach with only one corporate server, no replication or distribution of metadata or bulk data, but distributed clients, the main bottle neck will be the wide area network because for each transaction metadata and bulk data have to be transferred. This approach represents the easiest solution considering IT requirements but supplies inadequate response times in the case of high date volumes and frequent inquiries to the central system. Therefore, it makes sense within temporary distributed environments, e.g. virtual enterprises or during the 
pilot phase of a distributed engineering environment, but for a permanent connection of two distributed design departments it does not provide satisfactory performance. However, this approach is lately revalued since most PDM vendors provide with the latest system versions an Internet/WWW-link which enables a distributed access to a PDM system via Internet using a standard HTML browser.

The most frequent solution within actual industrial applications is the distribution of user data with a central metadata storage. In this case, the user data can be both replicated, i.e. physically duplicated, and fragmented, i.e. physically divided. Since metadata compared to bulk data have by far a smaller volume, such a configuration offers a sufficient performance, particularly with a limited number of sites in a static enterprise structure with a fast network link. Some systems additionally allow the distribution of the metadata on different database-servers, which leads to a further reduction in response times and in network load, but also to increased system complexity.

\subsection{Linked PDM}

The linking of autonomous software systems is the most common solution for the IT support of distributed processes. For the management of distributed data and processes each site relies on its own PDM system controlling their own data. In order to support processes at a single site the PDM systems act autonomously, but for cross-site processes they have to use data converters.

For the exchange of data between PDM systems respectively for the import and the export of PDM data, a series of international standards were successfully introduced in recent years, e.g. IGES, STEP, SGML, HTML, AP214ORG, ISO10007, ISO 13584 and OFTP (Abramovici, Gerhard, Langenberg 1997). Nevertheless, an uniform standard for the entire PDM data exchange is not yet available. The exchange of data between PDM systems occurs in accordance with VDA 4914 (ENGDAT) in the form of so-called data packages. A data package contains the model data or bulk data as well as the associated metadata and transport data. Although industrial solutions for the linking of homogenous PDM environments are already offered by software vendors, these concepts rely on proprietary exchange formats and interfaces. The linking of heterogeneous PDM systems, however, has to be implemented individually.

The main advantage of linked PDM systems is the complete adoption of all local IT environments, data models and workflows. The linking is implemented as an add-on to the existing PDM solution and it is activated only when data has to be exchanged between different sites. On the other side the user has to accept long delay times and the possibility of data redundancy between multiple sites which may lead to the use of obsolete or inconsistent information. In order to control redundancy and assure consistency among multiple sites all data transfers have to be permitted and logged by a central control system. 


\subsection{Federated PDM}

PDM systems of a federated engineering environment are autonomous resulting in a structure of equal PDM systems that co-operate on the basis of a common interface model. Such a solution links or integrates a pool of independent installations which are usually heterogeneous and employ different PDM data models. Queries or other client actions can either be processed directly by the local respectively internal PDM server or via the local server by a remote server in the federation network. The co-operation between the different environments is guaranteed by so-called federation server modules or federation services within each system. Accordingly to the federation of databases (Conrad 1997) federated PDM environments can be represented schematically as follows (Figure 3). PDM systems supporting federated environments are currently subject of current development efforts of some system vendors and partially already available, nonetheless only on the basis of one individual PDM-system.

Federated PDM-System

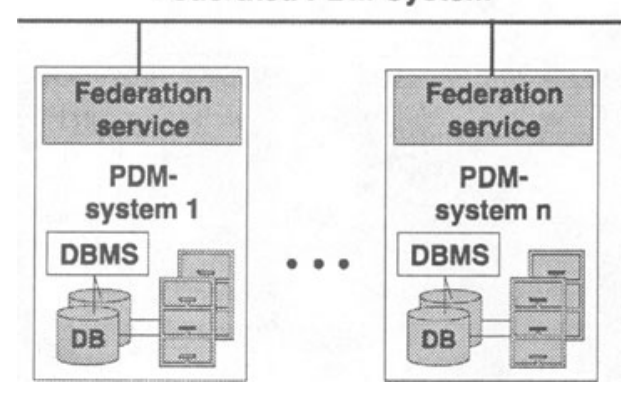

Figure 3 Federated PDM approach

Presently, the federation service of commercially available solutions for federated PDM only performs reading operations such as querying and copying of entities as well as creating and deleting of relations between entities. Another serious restriction of these systems is that they are usually based on a homogeneous environment where all sites or co-operating partners have to agree upon a uniform PDM-system. Modern forms of co-operation, like customersupplier-chains or virtual enterprises, however, in general do not meet the requirements of a homogeneous solution, but will provide a heterogeneous environment regarding the system, data model, process model and organisational level.

\section{INDUSTRIAL APPLICATIONS}

Currently, homogeneous distributed system environments are widely supported by commercially available systems. However, the majority of distributed PDM applications used productively within industry has been implemented as a user specific solution with a considerable expenditure for customising. Applications of geographically dispersed PDM-systems are predominantly used within larger 
enterprises. In the following two industrial case studies of a distributed and a linked PDM approach are presented. As the federated concept is currently subject of actual developments of PDM-vendors, an industrial application could not be found.

\section{Case Study: Distributed PDM}

An example of an industrial application of distributed PDM is the approach of Bosch-Siemens-Hausgeraete (BSHG), a world wide operating appliances manufacturer (Moebius 1997). In order to assure common access to product development data located at geographically dispersed sites, a company-wide uniform data model was specified. Moreover, the PDM system Metaphase has been introduced company-wide with the following distribution approach (Figure 4). Bulk data is fragmented distributed (document vaults) and the databases containing metadata are partially fragmented and partially replicated on the different sites in accordance to the Metaphase Distributed Architecture with a corporate server and subordinate workgroup servers and application servers.

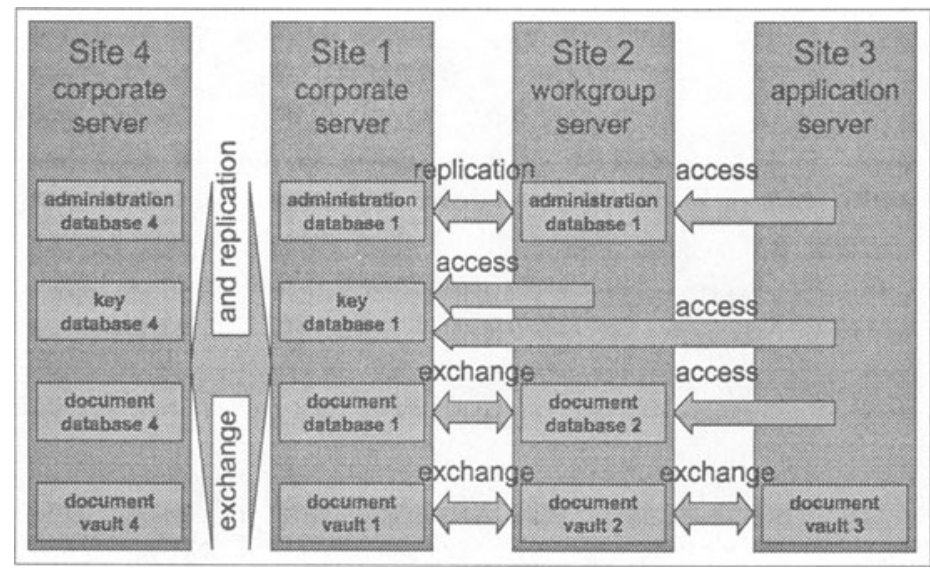

Figure 4 BSHG distributed PDM approach.(Moebius 1997)

This approach enables BSHG to store their product data in a distributed environment with controlled redundancy and to support thereby distributed product development processes. Other requirements for a PDM solution at BSHG are the capability to smoothly integrate into the existing IT system landscape, scalability of the PDM environment enabling a stepwise integration of further sites and the short response times during cross-site actions. Finally safety mechanisms and the comprehensibility of executed cross-site actions play a decisive role for industrial users. First experiences of this case and other industrial applications, however, show that the grown structures within the individual sites are not or only hardly to map through a uniform PDM data model even in the case of one single enterprise. 


\section{Case Study: Linked PDM}

A linked PDM approach seems best suited for heterogeneous multi-site development processes as found in customer-supplier-chains and virtual enterprises. A pilot application for the exchange of CAD-data and metadata including organisational and structural information was developed by MercedesBenz within the SWAN project (Kaschmieder, 1997). As the implementation of a homogeneous infrastructure with a uniform PDM data model between MercedesBenz and its suppliers within a customer-supplier-chain was not feasible an exchange management system was implemented. The exchange management system performs acknowledgement, logging of the exchange processes and filing of bulk data, organisational data and transport data according to ENGDAT. For safety reasons, access does not occur directly via PDM systems but via CAD systems whereas the direct course should be implemented in a next step.

First experiences of this project show the importance of a controlled import and export of data between different sites in order to enable recherche and recovery of access and exchange processes. Another crucial demand is the ability to implement safety, access protection and encryption mechanisms of exchange data according to bilateral defined agreements. When applying PDM technology security and authentication are best provided as a core part of the PDM data model. In order to ensure confidentiality and authenticity of all transferred model data reliable cryptographic techniques have to be integrated into the PDM solution, e.g. digital signature of CAD model changes.

\section{General evaluation}

In general, applications with multi-site exchange of organisational and structural data suffer the problem that available standards have not yet matured and have not yet been implemented consequently by all system vendors. Furthermore, in spite of all international standardisation efforts differences still exist between US American and European standards. In particular in the area of the exchange and of the semantic interpretation of metadata, the available standards meet the requirements inadequately.

Further problems arise due to the monolithic structure of commercially available PDM systems as each site requires its own functionality and user interfaces for the PDM system. By use of new modular PDM systems and configurable Java based graphical interfaces which are transferred to the user's workstation "on-demand", an optimised solution for the specific demands is provided. The above-mentioned functional requirements are not sufficiently covered by available default solutions but must be converted project-specifically with high customising expenditure. Especially safety mechanisms and the comprehensibility of executed cross-site actions play a decisive role to for industrial users. 


\section{INDUSTRY DRIVEN RESEARCH ACTIVITIES}

As the analysis of industrial requirements shows, there is further development necessary, especially to meet the following demands:

- capability to query in distributed databases and other information resources

- capability of an easy integration into existing IT-environments

- low effort for customisation and configuration

- controlled import and export of data

- ability of distributed data storage with a controlled redundancy and a maintenance of consistency

- ability to implement security and authentication mechanisms for confidential data

Several research activities deal with the development of extended PDM functionalities, mainly emphasising on flexibility and the integration of engineering marketplaces (Radeke, Seifert, 1998) (Zarli, Richaud, Amar, 1998). Another example is the Webflex-PDM approach currently under development at ITM, Bochum, is focussing on a web-enabled flexible PDM federation as an enhancement to existing federation concepts of vendors and other research institutes with special respect to a flexible and easy integration into existing heterogeneous IT-environments, the capability to query in distributed databases and other information ressources like engineering marketplaces, and low effort for customisation and configuration.

This research concepts is not based on the paradigm of a complete integration of different heterogeneous data resources in a distributed manner on semantic level but on a federated concept with a controlled and reconstructable redundancy. Especially in temporary limited co-operations the expenditures of a homogeneous PDM solution would by far outweigh its potential benefits in addition to the fact that the required time for setting up the environment would likely extend the time of co-operation within a project. Therefore flexible heterogeneous PDM solutions are required since approaches on the basis of a semantic integration on model level via the import and export of heterogeneous database schemes or through an automated mapping of information on semantic level have evidently not proven applicability due to the enormous complexity and the lack of flexibility. 


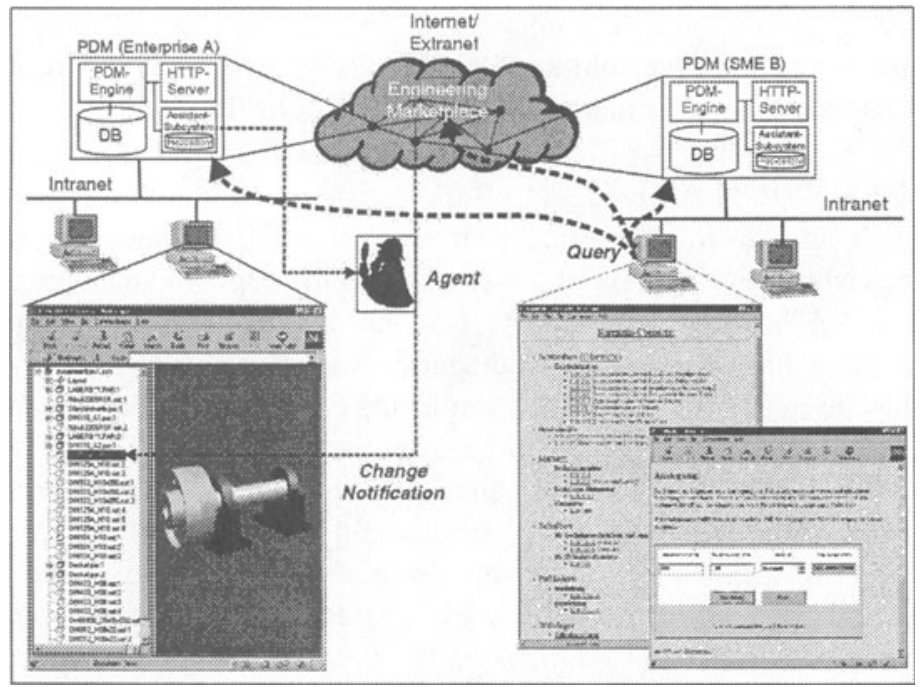

Figure 5 Web-enabled flexible PDM federations (Webflex-PDM)

The main goal of this approach is to optimally support the creativity and the competence of the users by enabling an effective co-operative use of distributed and heterogeneous data resources but leaving the semantic interpretation of data for decision making processes to the user. Webflex-PDM employs the use of agent technology and robot mechanisms and works as an assistant-system which intelligently supports users in database queries, data exchange and maintenance of data consistency by comprehending relations between different objects (Figure 5). Information of distributed IT systems with their relations are made transparently and available in a edited manner through suitable visualisation techniques, logging and history mechanisms as well as advanced retrieval techniques in order to represent the semantics and the complex relation networks between different data objects vividly and comprehensibly for the user.

According to this approach the PDM-system basically consists of a PDMengine, a built-in HTTP-Server to employ internet technology and an assistantsubsystem which also provides the federation service to allow multi-site queries for instance. For the implementation this approach requires the consequent usage of Internet technology, i.e. TCP/IP, CORBA, Java, HTML, VRML, which serves as a basic level of standardisation. Most of the available IT-systems within engineering environments offer interfaces to Internet technology. On this basis the adaption of legacy systems and the implementation of additional services can be achieved with comparatively less effort. As a result, an extensive independence is achieved from hardware and operating systems.

As PDM-technology is a rather pragmatic approach that evolved after the negative experiences in the implementation of the CIM vision, the Webflex-PDM concept tries to continue the pragmatism to extend PDM functionality and offers, 
from today's point of view, less integration, less automation but more flexibility which is not the appropriate solution for all kinds of distributed environments but especially suited for virtual enterprises or alliances of SME.

\section{CONCLUSION AND OUTLOOK}

PDM already is a well established technology for the management of data in single-site product development processes. Due to its pragmatic but comprehensive approach, PDM has the capability to become an enabling technology for the support of distributed product development processes, as well. Today's industrial applications are mainly homogeneous single-site environments but the demand for geographically dispersed co-operations forces the development of heterogeneous multi-site environments. Ongoing research and industrial activities try to bridge the gap between the demands and the functionality of available solutions. New approaches for distributed PDM-solutions are based on federated concepts. Due to market limitations vendors are mainly narrowed to single-system solutions, but research institutes, such as ITM, focus on the flexible and dynamic integration of heterogeneous multi-system multi-site environments.

One major step towards an overall IT support of product development processes concerning distributed and heterogeneous environments is the ViP project (Innovative Technologies and Systems for Virtual Product Development), funded by the German Federal Ministry of Education, Science, Research and Technology (BMBF). Within this project the ITM as a partner of the consortium is going to contribute to the achievement of this goal by further development and extension of the results and experiences of the introduced research work.

\section{REFERENCES}

Abramovici, M.; Gerhard D.; Langenberg, L. (1997) Application of PDM technology for Product Life Cycle Management, in: Krause, F.-L., Seliger, G. (Editors) Life Cycle Networks Proceedings of the 4th CIRP International Seminar on Life Cycle Engineering, June 26-27, 1997, Berlin, Chapman \& Hall, London, pp. 17-31.

Conrad, S. (1997): Foederierte Datenbanksysteme: Konzepte der Datenintegration, Springer, Berlin.

Kaschmieder, D. (1997) Einsatz des Datenaustauschmanagementsystems SWAN fuer den CAD-Datenaustausch mit Schnittstellen zu EDM-Systemen, in Proceedings Ploenzke EDM-Kongress, 23.-25.4.1997,Mainz.

Moebius, M.(1997) PDM at Bosch-Siemens Household Appliances (BSH), in Proceedings PDM Europe Conference 97, October 28-30, 1997, Noordwijk.

Radeke, E., Seifert, L. (1998) GENIAL: Enabling Intelligent Access to Internal and External Engineering Information, in Anderl, R., Encarnacao, J. L., Rix, J. (Editors) Tagungsband CAD '98 Tele-CAD Produktentwicklung in Netzwerken, Darmstadt, March 1998, pp. 75-84. 
Zarli, A., Richaud, O., Amar, V. (1998) An open flexible distributed architecture for the Large Scale Engineering, in European Conference Product Data Technology Days 1998, Proceedings, QMS, Sandhurst, UK, pp. 237-246.

\section{BIOGRAPHY}

Prof. Dr.-Ing. Michael Abramovici studied Mechanical Engineering at the Technical University of Berlin and received his doctor's degree in 1985. After holding different research and industrial positions he became Professor for Information Technology in Mechanical Engineering at the Ruhr-Universitaet Bochum in 1994.

Dipl.-Ing. Detlef Gerhard studied Mechanical Engineering at the University of Paderborn with a major in computer integrated production. Since 1995 he is working as research assistant at the Information Technology in Mechanical Engineering (ITM) division of the Ruhr-Universitaet Bochum.

Dipl.-Ing. Lars Langenberg studied Information Technology in Mechanical Engineering at the Technical University of Berlin. Since 1995 he is working as research assistant at the Information Technology in Mechanical Engineering (ITM) division of the RuhrUniversitaet Bochum. 